\title{
Malignant Peripheral Nerve Sheath Tumor with Perineurial Differentiation
}

National Cancer Institute

\section{Source}

National Cancer Institute. Malignant Peripheral Nerve Sheath Tumor with Perineurial

Differentiation. NCI Thesaurus. Code C66845.

A very rare malignant tumor with morphologic features similar to those of benign perineurioma of soft tissue along with hypercellularity, nuclear atypia, hyperchromasia, and a high mitotic rate. 